\title{
ON THE COMPLETENESS OF THE ROOT FUNCTIONS OF THE STURM-LIOUVILLE PROBLEMS FOR THE LAMÉ SYSTEM IN WEIGHTED SPACES
}

\author{
A. SHLAPUNOV AND A. PEICHEVA
}

\begin{abstract}
We consider three Sturm-Liouville boundary value problems (the coercive ones and the non-coercive one) in a bounded Lipschitz domain for the perturbed Lamé operator with the boundary conditions of Robin type. We prove that the problems are Fredholm ones in proper weighted Sobolev type spaces. The conditions, providing the completeness of the root functions related to the boundary value problem, are described.
\end{abstract}

\section{CONTEnTs}

1. Introduction

2. Function spaces

3. The Sturm Liouville-problem for the Lamé type system 3

4. The spectral properties of the mixed problems 12

References

\section{INTRODUCTION}

Investigating a boundary value problem, it is important to know both solvability conditions and formulas for its exact and approximate solutions. For the linear problems, the latter ones can be obtain with the use of expansions over the (generalized) eigenfunctions related to the them (see, for instance, [13]). Then, to use numerical methods in the non-selfadjoint case, one needs to prove the completeness of the system of the corresponding root elements. The results of this kind are well known for the coercive (elliptic) boundary problems over smooth domains (see [1, [7, 14]). For the Spectral Theory related to the elliptic problems in Lipschitz domains we refer to the survey [4. The root elements of general elliptic problems in weighted Sobolev spaces over domains with the conic and edge singularities were studied in [10, [16], 27].

Non-coercive boundary value problems for elliptic differential operators were discovered in the middle of XX-th century (see, 2], 15). In the Elasticity Theory, the problems of this kind were indicated in [8, 9]. Considering the non-coercive

2010 Mathematics Subject Classification. 35J48, 47A75.

Key words and phrases. Sturm-Liouville problem, non-coercive problems, the Lamé system, root functions.

THIS IS A PREPRINT VERSION OF THE PAPER PUBLISHED IN Z. ANGEW. MATH. MECH., V. 95, N. 11 (2015), 1202-1214. DOI 10.1002/ZAMM.201300303. 
problems, we essentially enlarge the class of boundary conditions for which the completeness of the root elements holds true. This may lead to a loss of the regularity of solutions to the problem near the boundary, but this is motivated by the very nature of the problems (cf. [23], 24]).

The aim of this paper is the proof of the completeness in weighted Sobolev type spaces of the root elements of three Sturm-Liouville problems for the perturbed Lamé operator with the boundary conditions of Robin type. The use of the weighted spaces allows us to choose the solutions with prescribed asymptotic behavior near the singular points of the boundary.

\section{FunCtion SPACES}

Let $D$ be a bounded domain in the Euclidean space $\mathbb{R}^{m}, m \geq 2$, with a Lipschitz boundary. We consider complex-valued functions defined over the domain $D$ and its closure $\bar{D}$. For $s \in \mathbb{Z}_{+}$and $M \subset \bar{D}$, denote by $C^{s}(\bar{D}, M)$ the set of $s$ times continuously differentiable functions over $\bar{D}$ vanishing in a neighborhood of $\bar{M}$. Let $C_{0}^{\infty}(D)$ be the space of smooth functions with compact supports in $D$. The Hölder class with the exponent $0<\alpha \leq 1$ over the set $M \subset \mathbb{R}^{m}$ we denote by $C^{0, \alpha}(M)$. We write $L^{q}(D)$ for the standard Lebesgue space $(1 \leq q \leq+\infty)$. We also write $H^{s}(D), s \in \mathbb{N}$, for the Sobolev space of functions with all the weak derivatives up to order $s$ belonging to $L^{2}(D)$. Let $H_{0}^{s}(D)$ stand for the closure of $C_{0}^{\infty}(D)$ in $H^{s}(D)$. For positive non-integer $s$ we denote by $H^{s}(D)$ the Sobolev-Slobodetskii space, see, for instance, 20.

Considering the spaces with negative smoothness we use the following standard construction. Let $H^{+}$and $H^{0}$ be complex Hilbert spaces with inner products $(\cdot, \cdot)_{+}$ and $(\cdot, \cdot)_{0}$ respectively. Assume that $H^{+}$is embedded continuously into $H^{0}$ and denote by $J_{0}: H^{+} \rightarrow H^{0}$ the corresponding embedding. Moreover, we assume that $H^{+}$is dense in $H^{0}$. Then let $H^{-}$stand for the completion of $H^{+}$with respect to the norm $\|u\|_{-}=\sup _{\substack{v \in H^{+} \\ v \neq 0}} \frac{\left|(v, u)_{0}\right|}{\|v\|_{+}}$.

The following lemma is well known (see, for instance, [22, §3]).

Lemma 2.1. The Banach space $H^{-}$is topologically isomorphic to the dual space $\left(H^{+}\right)^{\prime}$. Besides, the isomorphism is defined by the Hermitian form $\langle v, u\rangle=\lim _{\nu \rightarrow \infty}\left(v, u_{\nu}\right)_{0}$, $u \in H^{-}, v \in H^{+}$, where $\left\{u_{\nu}\right\}$ is a sequence in $H^{+}$converging to $u$ in $H^{-}$. Moreover, if the embedding $J_{0}: H^{+} \rightarrow H^{-}$is compact then the space $H^{0}$ is compactly embedded to $\mathrm{H}^{-}$.

Thus, $H^{-s}(D), s>0$, corresponds to $H^{-}$if $H^{0}=L^{2}(D), H^{+}=H^{s}(D)$. If $H^{0}=L^{2}(D), H^{+}=H_{0}^{s}(D)$ the space $H^{-}$will be denoted by $\tilde{H}^{-s}(D)$.

The weighted spaces appears naturally during the investigation of mixed boundary problems because the weight can be used to control the behavior of the solutions near the set where the boundary conditions change the character. Choose a closed set $Y$ on $\partial D$. In order to control the growth of functions near $Y$ we introduce the weighted spaces associated to $Y$. Assume that $\rho \in C(\bar{D})$ is a $C^{1}$-smooth function over $\bar{D} \backslash Y$ such that $0 \leq \rho(x) \leq 1, x \in \bar{D}, \frac{\partial \rho}{\partial x_{j}} \in L^{\infty}(D), 1 \leq j \leq m$ and $\rho(x)=0$ if and only if $x \in Y$. In particular, $\rho \equiv 1$ will correspond to the usual Sobolev spaces. If $Y \neq \emptyset$, then in typical situations, for domains with piece-wise smooth boundaries, the function $\rho(x)$ is the distance form the point $x \in \bar{D}$ to the singular set $Y \subset \partial D$. 
Now, for $\gamma \in \mathbb{R}, s=0$ and $s=1$ we introduce the weighted Sobolev spaces $H^{s, \gamma}(D)$ as the completion of $C^{s}(\bar{D}, Y)$ with respect to the norms, induced by the following scalar products:

$$
(u, v)_{H^{s, \gamma}(D)}=\sum_{|\alpha| \leq s}\left(\rho^{|\alpha|-\gamma-s} \partial^{\alpha} u, \rho^{|\alpha|-\gamma-s} \partial^{\alpha} v\right)_{L^{2}(D)}, s=0,1,
$$

(cf. [6, §1.7] for the localized situation where the weight is given in local coordinates near the singularity). Moreover, for $0<s<1$ we introduce the weighted Sobolev-Slobodetskii spaces as the completion of $C^{1}(\bar{D}, Y)$ with respect to the norms, induced by the following scalar product:

$$
(u, v)_{H^{s, \gamma}(D)}=(u, v)_{H^{0, \gamma+s}(D)}+\left(\rho^{-\gamma} u, \rho^{-\gamma} v\right)_{H^{s}(D)} .
$$

Similar fractional weighted spaces were considered in [17 for the localized situation.

As before, the weighted negative Sobolev-Slobodetskii space $H^{-s, \gamma}(D), 0<s \leq$ 1 , will be defined as the space $H^{-}$for $H^{0}=H^{0, \gamma}(D), H^{+}=H^{s, \gamma}(D)$.

Lemma 2.2. For each fixed $\gamma \in \mathbb{R}$ the space $H^{s, \gamma}(D)$ is compactly embedded into $H^{s^{\prime}, \gamma}(D)$, if $-1 \leq s^{\prime}<s \leq 1$. Moreover, if $1 / 2<s \leq 1$ the trace operator tr: $H^{s, \gamma}(D) \rightarrow H^{s-1 / 2, \gamma}(\partial D)$ is correctly defined and bounded.

Proof. The proof is standard. It is based on the Rellich-Kondrashov Theorem and the Trace Theorem for the usual Sobolev spaces (see [6, §1.7]).

Everywhere below, for a set $M \subset \bar{D}$ we denote by $H^{s, \gamma}(D, M)$ the completion of $C^{s}(\bar{D}, M \cup Y)$ in $H^{s, \gamma}(D)$. In particular, $H^{1}(D, \partial D)=H_{0}^{1}(D)$.

Besides, for a function space $\mathfrak{B}(D)$ over $D$ denote by $[\mathfrak{B}(D)]^{k}$ the space of $k$ vector functions $u$ with the components $u_{j} \in \mathfrak{B}(D)$. If $\mathfrak{B}(D)$ is a normed space then we endow the space $[\mathfrak{B}(D)]^{k}$ with the norm $\|u\|_{[\mathfrak{B}(D)]^{k}}=\left(\sum_{j=1}^{k}\left\|u_{j}\right\|_{\mathfrak{B}(D)}^{2}\right)^{1 / 2}$. Thus, $[\mathfrak{B}(D)]^{k}$ is a Hilbert space if the space $\mathfrak{B}(D)$ is a Hilbert one.

\section{The Sturm Liouville-Problem for the Lamé type System}

Fix an open connected set $S$ with piece-wise smooth boundary $\partial S$ on the hypersurface $\partial D$, a set $Y \subset \partial S$ and a weight $\rho$ associated with them. Denote by $\mathcal{L}$ the Lamé type operator in $\mathbb{R}^{m}$ :

$$
\mathcal{L}_{0}(x, \partial)=-\mu(x) I_{m} \Delta_{m}-(\lambda(x)+\mu(x)) \nabla_{m} \operatorname{div}_{m}
$$

where $I_{m}$ is the identity $(m \times m)$-matrix, $\Delta_{m}$ is the Laplace operator in $\mathbb{R}^{m}, \nabla_{m}$ is the gradient operator in $\mathbb{R}^{m}, \operatorname{div}_{m}$ is the divergence operator in $\mathbb{R}^{m}$, and $\mu, \lambda$ are real-valued functions from $L^{\infty}(D)$ such that $\mu \geq \kappa,(2 \mu+\lambda) \geq \kappa$ for a constant $\kappa>0$. If $m=3$ and $\mu \geq 0, \lambda \geq 0$ this operator plays an essential role in the description of the displacement of an elastic body under the load (see [12]). It also can be used as a linearization of the stationary version of the Navier-Stokes' type equations for viscous compressible fluid if the pressure is known (see [19, §15]).

Clearly, the Lamé type operator is strongly elliptic and, if the functions $\mu$, $\lambda$ belong to $C^{0,1}(D)$ then there is a formally non-negative self-adjoint operator $\mathcal{L}_{\mathfrak{D}}(x, \partial)=\mathfrak{D}^{*} \mathfrak{D}$ that differs from $\mathcal{L}_{0}(x, \partial)$ by the low order summands; here $\mathfrak{D}=\sum_{j=1}^{m} \mathfrak{D}_{j} \partial_{j}$ is a differential $(k \times m)$-matrix first order operator and $\mathfrak{D}^{*}$ is its formal adjoint one. Of course, there are many such operators $\mathfrak{D}$. To introduce three of them we denote by $M_{1} \otimes M_{2}$ the Kronecker product of matrices $M_{1}$ and 
$M_{2}$, by $\operatorname{rot}_{m}$ we denote $\left(\frac{\left(m^{2}-m\right)}{2} \times m\right)$-matrix operator with the lines $\vec{e}_{i} \frac{\partial}{\partial x_{j}}-\vec{e}_{j} \frac{\partial}{\partial x_{i}}$, $1 \leq i<j \leq m$, representing the vorticity (or the standard rotation operator for $m=2, m=3)$, and by $\mathbb{D}_{m}$ we denote $\left(\frac{\left(m^{2}+m\right)}{2} \times m\right)$-matrix operator with the lines $\sqrt{2} \vec{e}_{i} \frac{\partial}{\partial x_{i}}, 1 \leq i \leq m$, and $\vec{e}_{i} \frac{\partial}{\partial x_{j}}+\vec{e}_{j} \frac{\partial}{\partial x_{i}}$ with $1 \leq i<j \leq m$, representing the deformation (the strain). The we set:

$\mathfrak{D}^{(1)}=\left(\begin{array}{c}\sqrt{\mu} \mathbb{D}_{m} \\ \sqrt{\lambda} \operatorname{div}_{m}\end{array}\right), \quad \mathfrak{D}^{(2)}=\left(\begin{array}{c}\sqrt{\mu} \nabla_{m} \otimes I_{m} \\ \sqrt{\mu+\lambda} \operatorname{div}_{m},\end{array}\right), \quad \mathfrak{D}^{(3)}=\left(\begin{array}{l}\sqrt{\mu} \operatorname{rot}_{m} \\ \sqrt{2 \mu+\lambda} \operatorname{div}_{m}\end{array}\right)$,

here $\lambda \geq 0, k_{1}=\left(m^{2}+m\right) / 2+1$ for the first operator, $(\mu+\lambda) \geq 0, k_{2}=m^{2}+1$ for the second operator, and $(2 \mu+\lambda) \geq \kappa>0, k_{3}=\left(m^{2}-m\right) / 2+1$ for the third operator.

Thus, everywhere below we assume that $\mu, \lambda \in C^{0,1}(D) \cap L^{\infty}(D), \rho \nabla_{m} \mu, \rho \nabla_{m} \lambda \in$ $\left[L^{\infty}(D)\right]^{m}$.

Consider a $(m \times m)$-matrix linear differential operator $A$ in the domain $D$ associated with the operator $\mathcal{L}_{\mathfrak{D}}$, where $\mathfrak{D}$ is one of the operators $\mathfrak{D}^{(j)}, j=1,2,3$ :

$$
A u=\mathfrak{D}^{*} \mathfrak{D} u+a_{1} \nabla_{m} \otimes I_{m}+a_{0}(x) u,
$$

here $a_{0}$ and $a_{1}$ are functional $(m \times m)$ - and $\left(m \times m^{2}\right)$ - matrices respectively with the components $a_{j}^{(p, q)}$ satisfying the following assumptions: $\rho^{2} a_{0}^{(p, q)} \in L^{\infty}(D)$, $\rho a_{1}^{(p, q)} \in L^{\infty}(D)$.

Let $\nu_{\mathfrak{D}}=\sum_{j=1}^{m} \mathfrak{D}_{j}^{*} \nu_{j} \mathfrak{D}$ be the conormal derivative with respect to the operator $\mathfrak{D}$, where $\nu=\left(\nu_{1}, \ldots \nu_{m}\right)$ is the field of the exterior unit normal vectors with respect to $\partial D$ (defined for almost all points $x \in \partial D$ ). Clearly, two operators of the type $\nu_{\mathfrak{D}}$ above, differ on a matrix with entries being tangential derivatives with respect to the boundary.

Consider now the boundary operator

$$
B=b_{1}(x) \nu_{\mathfrak{D}}+b_{0}(x)+\partial_{\tau}
$$

where $\partial_{\tau}$ is a $(m \times m)$-matrix of tangential derivatives with respect to $\partial D$. As for the $(m \times m)$-matrices $b_{0}(x)$ and $b_{1}(x)$, we will assume that their components are locally bounded functions on $\partial D \backslash Y$. We allow for the matrix $b_{1}(x)$ to degenerate on $S$; in this case we assume that $b_{0}(x)$ is not degenerate on $S$ and the components of the tangential part $\partial_{\tau}$ equal to zero on $S$.

Remark 3.1. Usually, the first order boundary conditions related to boundary problems for the Lamé operator are defined with the use of the stress boundary tensor $\sigma$ with the components

$$
\sigma_{i, j}=\mu \delta_{i, j} \sum_{k=1}^{m} \nu_{k} \frac{\partial}{\partial x_{k}}+\mu \nu_{j} \frac{\partial}{\partial x_{i}}+\lambda \nu_{i} \frac{\partial}{\partial x_{j}}, 1 \leq i, j \leq m .
$$

Then, with the tangential part $\partial_{\tau_{0}}=\left(\left(\nu(x) \operatorname{div}_{\mathrm{m}}\right)^{\mathrm{T}}-\nu(\mathrm{x}) \operatorname{div}_{\mathrm{m}}\right)$, we have

$$
\sigma=\nu_{\mathfrak{D}^{(1)}}=\nu_{\mathfrak{D}^{(2)}}+\mu(x) \partial_{\tau_{0}}=\nu_{\mathfrak{D}^{(3)}}+2 \mu(x) \partial_{\tau_{0}} .
$$

We will study the following mixed problem: given generalized $m$-vector function $f$ in $D$, find a $m$-vector distribution $u$ in $D$ satisfying in a proper sense (cf. [12, $\S 12]$ for $\mathfrak{D}=\mathfrak{D}^{(1)}$ )

$$
\left\{\begin{array}{l}
A u=f \quad \text { in } \quad D \\
B u=0 \text { on } \quad \partial D .
\end{array}\right.
$$


If $S=\partial D$ then we obtain the classical Dirichlet problem for the strongly elliptic operator $\mathfrak{D}^{*} \mathfrak{D}$. As it is well known, it is coercive due to the Gårding inequality (see, for instance, 12, [18, 20]). That is why we will be concentrated on the case where $S \neq \partial D$.

The boundary problem (3.6) related to $\mathfrak{D}=\mathfrak{D}^{(3)}$ was discovered by S. Campanato (see [8], 9]). However he proved an Existence Theorem for it in the coercive case $S=\partial D$ only.

In the classical Theory of Boundary Value Problems, a typical assumptions are the fulfillment of the Shapiro-Lopatinsky conditions for the pair $(A, B)$ on the smooth part of $\partial D$ (see, for instance, [11, [18, 20, and others), that is a necessary for the problem to be coercive. We will show below that for $S \neq \partial D$ and $\mathfrak{D}=\mathfrak{D}^{(1)}$ or $\mathfrak{D}=\mathfrak{D}^{(2)}$ the mixed problem (3.6) is coercive in the Sobolev spaces, but for $S \neq \partial D$ and $\mathfrak{D}=\mathfrak{D}^{(3)}$ it is not (cf. $[\underline{8}$ for $n=2$ ).

As we plan to use the perturbation method for compact self-adjoint operators, we split the coefficients $a_{0}$ and $b_{0}$ :

$$
a_{0}=a_{0,0}+\delta a_{0}, b_{0}=b_{0,0}+\delta b_{0},
$$

where $a_{0,0}(x)$ is a Hermitian non-negative functional $(m \times m)$-matrix over $D$ with the components satisfying $\rho^{2} a_{0,0}^{(p, q)} \in L^{\infty}(D)$, and where $(m \times m)$-matrix $b_{0,0}$ is chosen in such a way that $b_{1}^{-1} b_{0,0}$ is Hermitian non-negative functional matrix over $\partial D$.

Consider the following Hermitian forms on the space $\left[H^{1}(D, S \cup Y)\right]^{m}$ :

$(u, v)_{+, \gamma, \mathfrak{D}(j)}=\left(\mathfrak{D}^{(j)} u, \mathfrak{D}^{(j)} v\right)_{\left[H^{0, \gamma}(D)\right]^{k}}+\left(a_{0,0} u, v\right)_{\left[H^{0, \gamma}(D)\right]^{m}}+\left(b_{1}^{-1} b_{0,0} u, v\right)_{\left[H^{0, \gamma}(\partial D \backslash S)\right]^{m}}$.

The form $(\cdot, \cdot)_{+, \gamma, \mathfrak{D}^{(2)}}$ is strongly coercive, i.e.

$$
\left\|\mathfrak{D}^{(2)} u\right\|_{\left[H^{0, \gamma}(D)\right]^{k_{1}}}^{2} \geq c\left\|\nabla_{m} u_{j}\right\|_{H^{0, \gamma}(D)}^{2} \text { for all } u \in\left[H^{1}(D)\right]^{m}
$$

with a constant $c$ being independent on $u$. The forms corresponding to operators $\mathfrak{D}^{(1)}$ and $\mathfrak{D}^{(3)}$ are not strongly coercive because $\mathfrak{D}^{(1)} u=0$ with non-constant vector $u=x_{i} \vec{e}_{j}-x_{j} \vec{e}_{i}, i \neq j$ and $\mathfrak{D}^{(3)} \nabla_{m} h=0$ in $D$ for any harmonic function $h$ in $D$.

Denote by $H_{\mathcal{D}^{(j)}}^{+, \gamma}(D), j=1,2,3$, the completion of $\left[H^{1}(D, S \cup Y)\right]^{k}$ with respect to the norm $\|\cdot\|_{+, \gamma, \mathfrak{D}^{(j)}}$ induced by the inner product $(\cdot, \cdot)_{+, \gamma, \mathfrak{D}^{(j)}}$ (of course, if it is an inner product).

Lemma 3.2. The Hermitian form $(\cdot, \cdot)_{+, \gamma, \mathfrak{D}^{(j)}}$ defines an inner product on $\left[H^{1}(D, S \cup\right.$ $Y)]^{m}$ if one of the following conditions holds true:

1) the open set $S \subset \partial D$ is not empty (in the topology of $\partial D$ );

2) $a_{0,0} \geq c_{0} I_{m}$ in $\bar{U}$ with a constant $c_{0}>0$ on a non-empty open set $U \subset D$;

3) $b_{1}^{-1} b_{0,0} \geq c_{1} I_{m}$ in $\bar{V}$ with a constant $c_{1}>0$ on a non-empty open set $V \subset$ $\partial D \backslash S$.

Besides, in these cases we have:

a) the space $\left[H^{1, \gamma}(D, S)\right]^{m}$ is continuously embedded in $H_{\mathfrak{D}}^{+, \gamma}(D)$ if the components of the matrix $\rho b_{1}^{-1} b_{0,0}$ belong to $L^{\infty}(\partial D \backslash S)$;

b) the elements of $H_{\mathcal{D}^{(j)}}^{+, \gamma}(D)$ belong to $\left[H_{\mathrm{loc}}^{1}(D \cup S, S)\right]^{m}$.

Proof. Regarding the statement on the scalar product, we only need to check that $(u, u)_{+, \gamma, \mathfrak{D}}=0$ for $u \in\left[H^{1}(D, S \cup Y)\right]^{m}$ implies $u=0$ in $D$. 
But the first order operators

$$
\begin{aligned}
\left(\begin{array}{cc}
\sqrt{\mu^{-1}} I_{k_{1}-1} & 0 \\
0 & \sqrt{\lambda^{-1}}
\end{array}\right) \mathfrak{D}^{(1)}, & \left(\begin{array}{cc}
\sqrt{\mu^{-1}} I_{k_{2}-1} & 0 \\
0 & \sqrt{(\mu+\lambda)^{-1}}
\end{array}\right) \mathfrak{D}^{(2)} \\
& \left(\begin{array}{cc}
\sqrt{\mu^{-1}} I_{k_{3}-1} & 0 \\
0 & \sqrt{(2 \mu+\lambda)^{-1}}
\end{array}\right) \mathfrak{D}^{(3)}
\end{aligned}
$$

have constant coefficients and injective principal symbols. Then Petrovskii Theorem implies that the distributions-solutions to $\mathfrak{D}^{(j)} u=0$ in $D, j=1,2,3$, are real analytic there. Hence the statement on the scalar product under condition 2) follows from the Uniqueness Theorem for real analytic functions.

Then it follows from conditions 1) or 3) that any vector $u \in\left[H^{1}(D, S \cup Y)\right]^{m}$ satisfying $(u, u)_{+, \gamma, \mathfrak{D}}=0$ vanishes on an open non-empty subset of $\partial D$. As $u$ also satisfies $\mathfrak{D}^{(j)} u=0$ in $D$, the Uniqueness Theorem for the Cauchy problem for systems with injective symbols implies that $u \equiv 0$ in $D$ (see, for instance, [25, Theorem 2.8]).

As the solutions of the system $\mathfrak{D}^{(j)} u=0$ in $D$ real analytic there, then there are no such solutions of the class $\left[H^{1}(D, \partial D)\right]^{m}$. Then it follows from the Gårding inequality for the Hermitian form $(\mathfrak{D} \cdot \mathfrak{D} \cdot)_{\left[L^{2}(D)\right]^{k}}$ induced by the strongly elliptic operator $\mathfrak{D}^{*} \mathfrak{D}$ that

$$
\|u\|_{\left[H^{1}(D)\right]^{m}}^{2} \leq \operatorname{const}(\mathfrak{D} u, \mathfrak{D} u)_{\left[L^{2}(D)\right]^{k}} \text { for all } u \in\left[H^{1}(D, \partial D)\right]^{m},
$$

the constant const being independent on $u$. In particular, this implies that the statement b) holds true

Finally, the statement a) can be checked directly.

Lemma 3.3. Let $\mu, \lambda \in L^{\infty}(D),(\mu+\lambda) \geq 0$. If $\rho \equiv 1$ then the embedding $H_{\mathfrak{O}(j)}^{+, \gamma}(D) \rightarrow\left[H^{1}(D)\right]^{m}, j=1,2$ is bounded under one of the conditions 1), 2), 3) of Lemma 3.2. If there is $q>0$ such that

$$
\rho^{2} a_{0,0} \geq q I_{m} \text { in } \bar{D} \backslash Y,
$$

then the space $H_{\mathfrak{D}^{(j)}}^{+, \gamma}(D)$ is continuously embedded into $\left[H^{1, \gamma}(D)\right]^{m}, j=1,2$.

Proof. The continuity of the embedding $H_{\mathfrak{O}^{(1)}}^{+, \gamma}(D) \rightarrow\left[H^{1, \gamma}(D)\right]^{m}$ follows from the second Korn inequality (see [12, formula (12.11)]). The continuity of the embedding $H_{\mathfrak{D}^{(2)}}^{+, \gamma}(D) \rightarrow\left[H^{1, \gamma}(D)\right]^{m}$ follows from the strong coercive estimate (3.7).

For the operator $\mathfrak{D}^{(3)}$ Lemma 3.3 is not true.

Example 3.4. Take the cylinder

$$
D=\left\{\left(x_{1}, x_{2}\right) \in \Omega, 0<x_{3}<1\right\}
$$

with the base $\Omega=\left\{x_{1}^{2}+x_{2}^{2}<1\right\}$ in $\mathbb{R}^{3}$ as the domain. Let, for instance, $\rho \equiv 1$, $a_{0,0} \equiv \rho^{-2}, b_{1}^{-1} b_{0,0} \in L^{\infty}(\partial D \backslash S)$, and $S=\emptyset$. Then $H^{1, \gamma}(D)=H^{1}(D)$. Set $h_{m}=\Re\left(x_{1}+\iota x_{2}\right)^{m}$, with $\iota$ being the imaginary unit and $\Re(a)$ being the real part of a complex number $a$. The function $h_{m}$ is harmonic in $D$ as the real part of the holomorphic monomial $\left(x_{1}+\iota x_{2}\right)^{m}$. It is easy to check that the system $\left\{\nabla_{3} h_{m}\right\}$ is orthogonal in $\left[H^{1}(\Omega)\right]^{3}$ and $\left[H^{1}(D)\right]^{3}$ (the last one follows from the Fubini Theorem). Then Bessel's Inequality implies that the sequence $\left\{u_{m}=\nabla_{3} h_{m} /\left\|\nabla_{3} h_{m}\right\|_{\left[H^{1}(D)\right]^{3}}\right\}$ converges weakly to zero in $\left[H^{1}(D)\right]^{3}$. It follows from the Sobolev Embedding Theorem that the sequence $\left\{u_{m}\right\}$ converges to zero $\left[L^{2}(D)\right]^{3}$ and $\left[L^{2}(\partial D)\right]^{3}$ while 
$\left\|u_{m}\right\|_{\left[H^{1}(D)\right]^{3}}=1$. Finally, as $\mathfrak{D}^{(3)} u_{m}=0$ we see that $\left\|u_{m}\right\|_{H_{\mathfrak{D}(3)}^{+, \gamma}(D)} \rightarrow 0$ if $m \rightarrow+\infty$. This means that the continuous embedding $H_{\mathfrak{D}(3)}^{+, \gamma}(D) \hookrightarrow\left[H^{1, \gamma}(D)\right]^{3}$ is impossible.

Example 3.5. In order to illustrate the case $S \neq \emptyset$ we set $m=2$. In this situation one can easily modify the famous Hadamard's example related to the ill-posed Cauchy for the Laplace operator. Namely, take the upper half-circle $\left\{x_{2}>0, x_{1}^{2}+\right.$ $\left.x_{2}^{2}<1\right\}$ as the domain $D$, and take the interval $[-1,1] \subset O x_{1}$ as the set $S$. For instance, let $a_{0,0} \equiv 0, b_{0,0} \equiv 0$ on $\left.\partial D \backslash S\right), \rho \equiv 1$. Note that the matrix ( $\left.\begin{array}{c}\operatorname{rot}_{2} \\ \operatorname{div}_{2}\end{array}\right)$ is adjoint to the Cauchy-Riemann system. On $\partial D$ we consider the sequence $\left\{v_{p}\right\}$ with the components

$$
v_{p}^{(1)}(x)=\left\{\begin{array}{ll}
\frac{1}{p} \sin \left(\pi p x_{1}\right), & x_{2}=0, \\
0, & x_{2}>0 .
\end{array} \quad, \quad v_{p}^{(2)} \equiv 0 .\right.
$$

Obviously, each $v_{p}$ is a Lipschitz function on $\partial D$ and the sequence $\left\{v_{p}\right\}$ converges to zero in $\left[H^{1 / 2}(\partial D)\right]^{2}$. If $P_{\Delta}$ stands for the Poisson integral for the Dirichlet Problem for the Laplace operator in $D$ then the sequence $\left\{P_{\Delta}\left(v_{m}\right)\right\}$ converges to zero in $\left[H^{1}(D)\right]^{2}$. Now it is clear that the functions

$$
\left\{u_{p}=\left(\begin{array}{c}
\Re\left(\sin \pi\left(x_{1}-\iota x_{2}\right)\right) \\
\Im\left(\sin \pi\left(x_{1}-\iota x_{2}\right)\right)
\end{array}\right)-P_{\Delta}\left(v_{p}\right)\right\}
$$

belong to $\left[H^{1}(D)\right]^{2}$ and they equal to zero on $S$ (here $\Im(a)$ denotes the imaginary part of a complex number $a$ ). Moreover, by the construction, the sequence $\left\{\mathfrak{D}^{(3)} u_{p}=-\mathfrak{D}^{(3)} P_{\Delta}\left(v_{p}\right)\right\}$ converges to zero in $L^{2}(D)$. That is why $\left\{u_{p}\right\}$ converges to zero in $H_{\mathfrak{D}^{(3)}}^{+, \gamma}(D)$ but it can not be convergent even in $\left[L^{2}(D)\right]^{2}$.

However, one can indicate conditions providing useful embedding theorems for the spaces generated by non-coercive forms (see [2]). The following statement describes reasonable assumptions for $H_{\mathcal{D}^{(3)}}^{+, \gamma}(D)$ to be embedded into Sobolev-Slobodetskii spaces. The scheme of its proof is similar to the cases of scalar operators (see [23, [24]).

Theorem 3.6. Let $\mu, \lambda$ belong to the class $C^{\infty}(X)$ with a neighborhood $X$ of the compact $\bar{D}$, and let $\rho \equiv 1$. Then

1) the space $H_{\mathfrak{D}^{(3)}}^{+, \gamma}(D)$ is continuously embedded into $\left[L^{2}(D)\right]^{m}$, if condition (3.9) holds true.

2) the space $H_{\mathfrak{D}(3)}^{+, \gamma}(D)$ is continuously embedded into $\left[H^{1 / 2-\varepsilon}(D)\right]^{m}$ with any $\varepsilon>0$ if

$$
b_{1}^{-1} b_{0,0} \geq c_{1} I_{m} \text { on } \partial D \backslash S \text { with a constant } c_{1}>0 .
$$

Moreover, if $\partial D \in C^{2}$, then (3.10) implies that the space $H_{\mathfrak{D}^{(3)}}^{+, \gamma}(D)$ is continuously embedded into $\left[H^{1 / 2}(D)\right]^{m}$.

Proof. The statement 1) is obviously true.

Let estimate (3.10) is fulfilled. Then the norm $\|\cdot\|_{+, \gamma, \mathfrak{D}^{(3)}}$ is not weaker than the norm $\|\cdot\|_{h}$ on $\left[H^{1}(D, S)\right]^{m}$, where

$$
\|u\|_{h}=\left(\left\|\mathfrak{D}^{(3)} u\right\|_{\left[L^{2}(D)\right]^{k}}^{2}+\|u\|_{\left[L^{2}(\partial D \backslash S)\right]^{m}}^{2}\right)^{1 / 2}, u \in\left[H^{1}(D, S)\right]^{m} .
$$

Fix a number $\varepsilon>0$. Let us show that the norm $\|\cdot\|_{h}$ is not weaker than the norm $\|\cdot\|_{\left[H^{1 / 2-\varepsilon}(D)\right]^{m}}$ on $\left[H^{1}(D, S)\right]^{k}$. Indeed, integrating by parts it is easy to 
see that a vector function $v \in\left[C^{\infty}(X)_{0}\right]^{m}$ satisfy $\left(\mathfrak{D}^{(3)}\right)^{*} \mathfrak{D}^{(3)} v=0$ in $X$ if and only if $\mathfrak{D}^{(3)} v=0$ in $X$. As we have seen in the proof of Lemma 3.2, any weak solution to this equation is real analytic in $X$ and hence we have $v \equiv 0$. Thus, under hypothesis of the theorem the operator $\mathcal{L}_{\mathfrak{D}^{(3)}}$ has a two-sided fundamental solution on $X$, say, $\phi_{m}(x, y)$. For instance, if $\mu$ and $\lambda$ are constants, we may take the famous Kelvin-Somigliana kernel.

The volume potential

$$
\Phi v(x)=\int_{D} \phi_{m}(x, y) v(y) d x, v \in\left[L^{2}(D)\right]^{m},
$$

induces the bounded linear operator $\Phi:\left[L^{2}(D)\right]^{m} \rightarrow\left[H^{2}(X)\right]^{m}$ for any bounded domain $X$ containing $\bar{D}$.

It is clear that any element $u \in H^{-s}(D)$ extends up to an element $U \in H^{-s}\left(\mathbb{R}^{m}\right)$ via

$$
\langle U, v\rangle_{\mathbb{R}^{m}}=\langle u, v\rangle_{D} \text { for all } v \in H^{s}\left(\mathbb{R}^{m}\right)
$$

here $\langle\cdot, \cdot\rangle_{D}$ is the pairing on $H \times H^{\prime}$ for a space $H$ of distributions over $D$. It is natural to denote it by $\chi_{D} u$. The defined in this way linear operator $\chi_{D}$ : $H^{-s}(D) \rightarrow H^{-s}\left(\mathbb{R}^{m}\right), s \in \mathbb{R}_{+}$is obviously bounded. Since the distribution $\chi_{D} u$ is supported in $\bar{D}$, the volume potential (3.12) induces the bounded linear operator

$$
\Phi \circ \chi_{D} I_{m}:\left[H^{\varepsilon-1 / 2}(D)\right]^{m} \rightarrow\left[H^{\varepsilon+3 / 2}(X)\right]^{m}, \quad 0<\varepsilon \leq 1 / 2,
$$

for any bounded domain $X$ containing $\bar{D}$ (see, [3]).

Hence, the operators

$$
\begin{gathered}
\mathfrak{D} \circ \Phi \circ \chi_{D} I_{m}:\left[H^{\varepsilon-1 / 2}(D)\right]^{m} \rightarrow\left[H^{\varepsilon+1 / 2}(X)\right]^{k}, \\
\nu_{\mathfrak{D}} \circ \Phi \circ \chi_{D} I_{m}:\left[H^{\varepsilon-1 / 2}(D)\right]^{m} \rightarrow\left[H^{\varepsilon}(\partial D)\right]^{m}
\end{gathered}
$$

are bounded, too, if $0<\varepsilon \leq 1 / 2$ because of the Trace Theorem for the Sobolev spaces. Note that for $\varepsilon=0$ this statement is not true because the elements of the space $H^{1 / 2}(X)$ may have no traces on $\partial D \subset X$.

Now integrating by parts we obtain for $u \in\left[H^{1}(D, S)\right]^{m}$ and $v \in\left[L^{2}(D)\right]^{m}$ :

$$
(v, u)_{\left[L^{2}(D)\right]^{m}}=\left(\mathcal{L}_{\mathfrak{D}} \Phi I_{m} v, u\right)_{\left[L^{2}(D)\right]^{m}}=\left(\mathfrak{D} \Phi I_{m} v, \mathfrak{D} u\right)_{\left[L^{2}(D)\right]^{k}}+\left(\nu_{\mathfrak{D}} \Phi I_{m} v, u\right)_{\left[L^{2}(\partial D \backslash S)\right]^{m}} .
$$

Take a sequence $\left\{v_{\mu}\right\} \subset\left[H^{1}(D)\right]^{m}$, converging to $v$ in the space $\left[H^{\varepsilon-1 / 2}(D)\right]^{m}$, $0<\varepsilon<1 / 2$. As the space $H^{s}(D)$ is reflexive for each $s$, using (3.13) and the continuity of the operators $\mathfrak{D} \circ \Phi \circ \chi_{D} I_{m}, \nu_{\mathfrak{D}} \circ \Phi \circ \chi_{D} I_{m}$ above, we obtain for $u \in\left[H^{1}(D, S)\right]^{m}:$

$c\|u\|_{\left[H^{1 / 2-\varepsilon}(D)\right]^{m}} \leq\left\|\mathfrak{D} \circ \Phi \circ \chi_{D} I_{m}\right\|\|\mathfrak{D} u\|_{\left[L^{2}(D)\right]^{k}}+\left\|\nu_{\mathfrak{D}} \circ \Phi \circ \chi_{D} I_{m}\right\|\|u\|_{\left[L^{2}(\partial D \backslash S)\right]^{m}}$ with a constant $c>0$ being independent on $u$. Thus, there are constant $C_{1}>0$, $C_{2}>0$ such that

$$
\|u\|_{\left[H^{1 / 2-\varepsilon}(D)\right]^{m}} \leq C_{1}\|u\|_{h} \leq C_{2}\|u\|_{+, \gamma} \text { for all } u \in\left[H^{1}(\bar{D}, S)\right]^{m} .
$$

This proves the continuous embedding $H^{+, \gamma}(D) \hookrightarrow\left[H^{1 / 2-\varepsilon}(D)\right]^{m}$ with any $\varepsilon>0$.

Due to the factorization, the operator $\mathcal{L}_{\mathfrak{D}}$ is strongly elliptic formally-selfadjoint and the Dirichlet problem for it is Fredholm of index zero (see, for instance, [5], 26. Lemma 3.2]). As we noted above, $\mathcal{L}_{\mathfrak{D}^{(3)}} u=0$ in $D$ for $u \in C_{0}^{\infty}(D)$ if and only if $u \equiv 0$. Therefore the Dirichlet problem for it is uniquely solvable. Let now $G$ and $P$ stand for the Green function and the Poisson integral of the Dirichlet Problem 
for the Dirichlet problem for the operator $\mathcal{L}_{\mathfrak{D}}$ in $D$. Then they induce the bounded operators (see, for instance, [5, 26, Theorem 3.3])

$$
G_{1}:\left[\tilde{H}^{-1}(D)\right]^{m} \rightarrow\left[H_{0}^{1}(D)\right]^{m}, \quad P_{1}:\left[H^{1 / 2}(D)\right]^{m} \rightarrow\left[H^{1}(D)\right]^{m} .
$$

As the operator $\mathcal{L}_{\mathfrak{D}}$ extends to the continuous linear operator $\mathcal{L}_{\mathfrak{D}}:\left[H^{1}(D)\right]^{m} \rightarrow$ $\left[\tilde{H}^{-1}(D)\right]^{m}$ via

$$
\left\langle\mathcal{L}_{\mathfrak{D}} u, v\right\rangle=(\mathfrak{D} u, \mathfrak{D} v)_{\left[L^{2}(D)\right]^{m}}, u \in\left[H^{1}(D)\right]^{m}, v \in\left[H_{0}^{1}(D)\right]^{m},
$$

then $u=P_{1} u+G_{1} \mathcal{L}_{\mathfrak{D}} u$ for each $u \in\left[H^{1}(D)\right]^{m}$. Hence, for $u, v \in\left[H^{1}(D, S)\right]^{m}$ we have:

$$
(u, v)_{h}=(P u, P v)_{\left[L^{2}(\partial D \backslash S)\right]^{m}}+\left(\mathfrak{D}^{(3)} u, \mathfrak{D}^{(3)} v\right)_{\left[L^{2}(D)\right]^{k}} .
$$

On the other hand, integrating by parts, we obtain

$$
\left(\mathfrak{D}^{(3)} P_{1} u, \mathfrak{D}^{(3)} G_{1} \mathcal{L}_{\mathfrak{D}^{(3)}} u\right)_{\left[L^{2}(D)\right]^{k}}=0 .
$$

That is why, for all $u \in\left[H^{1}(\bar{D}, S)\right]^{m}$,

$$
C_{2}^{2} C_{1}^{-2}\|u\|_{+, \gamma}^{2} \geq\|u\|_{h}^{2} \geq\left\|P_{1} u\right\|_{\left[L^{2}(\partial D \backslash S)\right]^{m}}^{2}+\left\|\mathfrak{D}^{(3)} G_{1} \mathcal{L}_{0} u\right\|_{\left[L^{2}(D)\right]^{k}}^{2}
$$

It follows from(3.8) and (3.14) that any sequence $\left\{u_{\mu}\right\} \subset\left[H^{1}(D, S)\right]^{m}$, converging to $u \in H_{\mathfrak{D}^{(3)}}^{+, \gamma}(D)$ in the space $H_{\mathfrak{D}^{(3)}}^{+, \gamma}(D)$ can be presented as

$$
u_{\mu}=P_{1} u_{\mu}+G_{1} \mathcal{L}_{\mathfrak{D}^{(3)}} u_{\mu}
$$

where the sequence $\left\{G_{1} \mathcal{L}_{\mathfrak{D}^{(3)}} u_{\mu}\right\}$ converges in $\left[H_{0}^{1}(D)\right]^{m} \subset\left[H^{1}(D, S)\right]^{m}$ to an element $w_{1}$.

Now the already proved part of the theorem yields that $\left\{P_{1} u_{\mu}\right\}$ converges to an element $w_{2}$ in $\left[H^{1 / 2-\varepsilon}(D)\right]^{m}$. This proves the continuous embedding $H_{\mathfrak{D}^{(3)}}^{+, \gamma}(D) \hookrightarrow$ $\left[H^{1 / 2-\varepsilon}(D)\right]^{m}$.

To finish the proof of the theorem one has to almost literally repeat the corresponding arguments in the proof of [23, Theorem 1], related to the mixed problem for the Laplace operator.

Corollary 3.7. Let $\mu, \lambda \in C^{\infty}(X)$ and estimates (3.9), (3.10) hold true. Then $H_{\mathcal{D}^{(3)}}^{+, \gamma}(D)$ is continuously embedded into $\left[H^{1 / 2-\varepsilon, \gamma}(D)\right]^{m}$ for any $\varepsilon>0$.

The embeddings, described in Theorem 3.6 and Corollary 3.7. are rather sharp on the scale of the Sobolev-Slobodetskii spaces (see Example 4.5 below).

Remark 3.8. Lemma 3.3. Theorem 3.6 and Corollary 3.7 imply that in the spaces $H_{\mathfrak{D}^{(1)}}^{+, \gamma}(D)$ and $H_{\mathfrak{D}^{(2)}}^{+, \gamma}(D)$ we can use arbitrary first order perturbations $a_{1} \nabla_{m} \otimes I_{m}$ in (3.3) with $\rho a_{1}^{(p, q)} \in L^{\infty}(D)$ while for the operators $\mathfrak{D}^{(3)}$ only the summands of the type $\tilde{a}_{1}(x) \mathfrak{D}^{(3)}$, where $\tilde{a}_{1}$ is a $\left(m \times k_{3}\right)$-matrix with entries $\tilde{a}_{1}^{(p, q)}$ satisfying $\rho \tilde{a}_{1}^{(p, q)} \in L^{\infty}(D)$, can be used.

Now we proceed with the generalized formulation of the Sturm-Liouville Problem. With this aim, we assume that $H_{\mathfrak{D}}^{+, \gamma}(D)=H^{+}$is continuously embedded into $H^{0, \gamma}(D)=H^{0}$ (the corresponding conditions were described above) and we denote by $H_{\mathfrak{O}}^{-, \gamma}(D)=H^{-}$the completeness of the space $\left[H^{1}(D, S)\right]^{m}$ with respect to the corresponding negative norm $\|u\|_{-, \gamma, \mathfrak{D}}$. The pairing, described in Lemma 2.1, will be denoted by $\langle\cdot, \cdot\rangle_{\gamma}$.

Further, on integrating by parts we see that

$$
(A u, v)_{\left[H^{0, \gamma}(D)\right]^{m}}=(\mathfrak{D} u, \mathfrak{D} v)_{\left[H^{0, \gamma}(D)\right]^{k}}+\left(b_{1}^{-1}\left(b_{0}+\partial_{\tau}\right) u, v\right)_{\left[H^{0, \gamma}(\partial D \backslash S)\right]^{m}}+
$$




$$
\left(a_{1} \nabla_{m} \otimes I_{m} u-2 \gamma \rho^{-1}(\mathfrak{D} \rho)^{*} \mathfrak{D} u+a_{0} u, v\right)_{\left[H^{0, \gamma}(D)\right]^{m}}
$$

for all $u \in\left[H^{2}(D, S)\right]^{m}$ and $v \in\left[H^{1}(D, S)\right]^{m}$, satisfying the boundary condition of (3.6); here $\mathfrak{D} \rho$ stands for the functional matrix $\sum_{j=1}^{m} \mathfrak{D}_{j} \frac{\partial \rho}{\partial x_{j}}$. Suppose that (cf. Remark 3.8)

$$
\left|\left(b_{1}^{-1}\left(\delta b_{0}+\partial_{\tau}\right) u, v\right)_{\left[L^{2}(\partial D \backslash S)\right]^{m}}+\left(a_{1} \nabla_{m} \otimes I_{m} u+\delta a_{0} u, v\right)_{\left[L^{2}(D)\right]^{m}}\right| \leq c\|u\|_{+, \gamma, \mathfrak{D}}\|v\|_{+, \gamma, \mathfrak{D}}
$$

for all $u, v \in\left[H^{1}(D, S \cup S)\right]^{m}$, with a positive constant $c$ being independent of $u$ and $v$.

Under condition (3.15), for each fixed $u \in H_{\mathfrak{D}}^{+, \gamma}(D)$ the sesquilinear form

$$
\begin{gathered}
Q(u, v)=(\mathfrak{D} u, \mathfrak{D} v)_{\left[H^{0, \gamma}(D)\right]^{l}}+\left(b_{1}^{-1} b_{0} u, v\right)_{\left[H^{0, \gamma}(\partial D \backslash S)\right]^{m}}+ \\
\left(a_{1} \nabla_{m} \otimes I_{m} u-2 \gamma \rho^{-1}(\mathfrak{D} \rho)^{*} \mathfrak{D} u+a_{0} u, v\right)_{\left[H^{0, \gamma}(D)\right]^{k}}
\end{gathered}
$$

determines a continuous linear functional $f$ on $H^{+, \gamma}(D)$ via the equality $f(v):=$ $\overline{Q(u, v)}$ for $v \in H^{+, \gamma}(D)$. By Lemma 2.1, there is a unique element $L u$ in $H^{-, \gamma}(D)$ such that

$$
f(v)=\langle v, L u\rangle_{\gamma}
$$

for all $v \in H^{+, \gamma}(D)$. We have thus defined a linear operator $L: H^{+, \gamma}(D) \rightarrow$ $H^{-, \gamma}(D)$. It follows from (3.15) that the operator $L$ is bounded. The bounded linear operator $L_{0}: H^{+, \gamma}(D) \rightarrow H^{-, \gamma}(D)$ defined in this way via the sesquilinear form $(\cdot, \cdot)_{+, \gamma}$, i.e.,

$$
(v, u)_{+, \gamma, \mathfrak{O}}=\left\langle v, L_{0} u\right\rangle_{\gamma}
$$

for all $u, v \in H_{+, \gamma}(D)$, corresponds to the case $a_{1}=\rho^{-1} \mathfrak{D}^{*} \rho, a_{0}=a_{0,0}$ and $b_{0}=b_{0,0}$.

Thus, the generalized setting of the problem (3.6) in the weighted spaces is the following: given $f \in H^{-, \gamma}(D)$ find $u \in H^{+, \gamma}(D)$ such that

$$
\overline{Q(u, v)}=\langle v, f\rangle_{\gamma} \text { for all } v \in H^{+, \gamma}(D) .
$$

The problem (3.17) can be investigated by the standard methods of functional analysis [18, Ch. 3, $\S \S 4-6])$ that are similar to the coercive case.

Lemma 3.9. Suppose that $H_{\mathfrak{D}}^{+, \gamma}(D)$ is continuously embedded into $H^{0, \gamma}(D), a_{1}=$ $2 \gamma \rho^{-1}(\mathfrak{D} \rho)^{*}, \delta a_{0}=0$ and $\delta b_{0}=0$. Then for each $f \in H_{\mathfrak{D}}^{-, \gamma}(D)$ there is a unique solution $u \in H_{\mathfrak{D}}^{+, \gamma}(D)$ to problem (3.17), i.e., the operator $L_{0}: H_{\mathfrak{D}}^{+, \gamma}(D) \rightarrow H_{\mathfrak{D}}^{-, \gamma}(D)$ is continuously invertible. Moreover, the norms of the operators $L_{0}$ and $L_{0}^{-1}$ equal to 1.

The following three lemmas describe bounded and compact perturbations of the operator $L_{0}$.

Lemma 3.10. Let $H_{\mathfrak{D}}^{+, \gamma}(D)$ be continuously embedded into $H^{s, \gamma}(D), 0<s \leq 1$. If $\rho a_{1} \in L^{\infty}(D), \rho^{2} \delta a_{0} \in L^{\infty}(D)$ then the corresponding summands in problem (3.17) induce bounded operators, acting from $H_{\mathfrak{D}}^{+, \gamma}(D)$ to $H_{\mathfrak{D}}^{-, \gamma}(D)$. Moreover, if there is $\varepsilon>0$ such that $\rho^{2-\varepsilon} \delta a_{0} \in L^{\infty}(D), \rho^{1-\varepsilon} \delta a_{1} \in L^{\infty}(D)$ then the corresponding summands in problem 3.17 induce compact operators, acting from from $H_{\mathfrak{D}}^{+, \gamma}(D)$ to $H_{\mathfrak{D}}^{-, \gamma}(D)$.

Proof. It follows from Lemma 2.2 
In the coercive case (corresponding to the operators $\mathfrak{D}^{(1)}, \mathfrak{D}^{(2)}$ ) we can enlarge the class of the perturbations. With this purpose we fix a basis $\left\{t_{j}\right\}_{j=1}^{m-1}$ among the tangential vectors (with bounded integrable components). For instance, this may be formed by the vectors

$$
\vec{e}_{j} \nu_{i}-\vec{e}_{i} \nu_{j}, \quad i>j
$$

Then $\partial_{\tau}=\sum_{j=1}^{m-1} d_{j}(x) \partial_{t_{j}}$ with $(m \times m)$-matrices $d_{j}(x)$.

Lemma 3.11. Let $j=1$ or $j=2$. Let (3.9) hold or $\rho \equiv 1$. If $\rho b_{1}^{-1} \delta b_{0} \in L^{\infty}(\partial D \backslash S)$ then the corresponding summand in problem (3.17) induces a bounded operator, acting from $H_{\mathfrak{D}^{(j)}}^{+, \gamma}(D)$ to $H_{\mathfrak{D}^{(j)}}^{-, \gamma}(D)$. if there is $\varepsilon>0$ such that $\rho^{1-\varepsilon} b_{1}^{-1} \delta b_{0} \in$ $L^{\infty}(\partial D \backslash S)$ then the corresponding summand in problem (3.17) induces a compact operator, acting from $H_{\mathfrak{D}^{(j)}}^{+, \gamma}(D) \rightarrow H_{\mathfrak{D}^{(j)}}^{-, \gamma}(D)$. Moreover, if $b_{1}^{-1} d_{j} \in C^{0, \alpha}(\partial D \backslash S)$, $1 \leq j \leq m-1,1 / 2<\alpha \leq 1$ then the matrix $\partial_{\tau}$ of tangential derivatives induces a bounded operator, acting from $H_{\mathfrak{D}^{(j)}}^{+, \gamma}(D)$ to $H_{\mathfrak{D}^{(j)}}^{-, \gamma}(D)$ with the norm estimated via $\left\|b_{1}^{-1} d_{j}\right\|_{C^{0, \alpha}(\partial D \backslash S)}, 1 \leq j \leq m-1$.

Proof. The continuity and the compactness of the operators induced by the summand $b_{1}^{-1} \delta b_{0}$ follows from Lemma 3.3, the Embedding Theorem for Sobolev spaces and the continuity of the trace operator $\operatorname{tr}: H^{1, \gamma}(D) \rightarrow H^{1 / 2, \gamma}(\partial D)$ (see Lemma 2.2).

In order to finish the proof of the continuity of the tangential operator one has to almost literally repeat the corresponding arguments in the proof of [24, Lemma $6.6]$, related to the similar mixed problem for the scalar differential operators.

Lemma 3.12. Let inequality (3.10) be fulfilled and $b_{1}^{-1} \delta b_{0} \in L^{\infty}(\partial D \backslash S)$. If (3.9) is true or $\rho \equiv 1$ then the corresponding summand in problem (3.17) induces a bounded operator, acting from $H_{\mathfrak{D}^{(3)}}^{+, \gamma}(D)$ to $H_{\mathfrak{D}^{(3)}}^{-, \gamma}(D)$.

As examples [23, Examples 1,2] show, the boundary terms $\delta b_{0}$ and $\partial_{\tau}$ do not induce compact and bounded perturbations respectively for $L_{0}$ if $m=2$ and $\mathfrak{D}=$ $\mathfrak{D}^{(3)}$.

Now we split

$\delta b_{0}=\delta b_{0}^{(s)}+\delta b_{0}^{(c)}, \quad \delta a_{0}=\delta a_{0}^{(s)}+\delta a_{0}^{(c)}, \quad a_{1} \nabla_{m} \otimes I_{m}=2 \gamma \rho^{-1}(\mathfrak{D} \rho)^{*} \mathfrak{D}+\left(\delta a_{1}^{(s)}+\delta a_{1}^{(c)}\right) \nabla_{m} \otimes I_{m}$ in such a way that the terms $\delta b_{0}^{(c)}, \delta a_{0}^{(c)}$ and $\delta a_{1}^{(c)}$ induce the compact perturbations of the operator $L_{0}$ and the summands $\delta b_{0}^{(s)}, \delta a_{0}^{(s)}$ and $\delta a_{1}^{(s)}$ induce the small ones. This gives the possibility to use the perturbation methods.

The proof of the following two statements is standard (see, for example, 20], 21], 24]).

Theorem 3.13. Let $j=1$ or $j=2$. Let $d_{j} \in C^{0, \lambda}(\partial D \backslash S), 1 \leq j \leq m-1$. Besides, let (3.9) hold or $\rho \equiv 1$. If there exists $\varepsilon>0$ such that $\rho^{2-\varepsilon} \delta a_{0}^{(c)} \in L^{\infty}(D)$, $\rho^{1-\varepsilon} \delta a_{1}^{(c)} \in L^{\infty}(D), \rho^{1-\varepsilon} \delta b_{0}^{(c)} \in L^{\infty}(\partial D \backslash S)$, and $\left|\left(b_{1}^{-1}\left(\delta b_{0}^{(s)}+\partial_{\tau}\right) u, v\right)_{\left[L^{2}(\partial D \backslash S)\right]^{m}}+\left(\delta a_{1}^{(s)} \nabla_{m} \otimes I_{m} u+\delta a_{0}^{(s)} u, v\right)_{\left[L^{2}(D)\right]^{m}}\right| \leq M\|u\|_{+, \gamma, \mathfrak{D}(j)}\|v\|_{+, \gamma, \mathfrak{D}^{(j)}}$

for all $u, v \in\left[H^{1}(D, S \cup S)\right]^{m}$ with a constant $0<M<1$ being independent on $u$ and $v$ then problem (3.17) is a Fredholm one.

For $\mathfrak{D}^{(3)}$ we split in a different way: $\tilde{a}_{1}=2 \gamma \rho^{-1}\left(\mathfrak{D}^{(3)} \rho\right)^{*}+\tilde{\delta} a_{1}^{(s)}+\delta \tilde{a}_{1}^{(c)}(\mathrm{cf}$. Remark 3.8). 
Theorem 3.14. Let $\mathfrak{D}=\mathfrak{D}^{(3)}$, estimate (3.10) hold, $\lambda, \mu$ are infinitely smooth in a neighborhood of $\bar{D}, \tau=0$ and $\delta b_{0}^{(c)}=0$. Besides, let (3.9) hold or $\rho \equiv 1$. If there exists $\varepsilon>0$ such that $\rho^{2-\varepsilon} \delta a_{0}^{(c)} \in L^{\infty}(D), \rho^{1-\varepsilon} \delta \tilde{a}_{1}^{(c)} \in L^{\infty}(D)$, and $\left|\left(b_{1}^{-1} \delta b_{0}^{(s)} u, v\right)_{\left[L^{2}(\partial D \backslash S)\right]^{m}}+\left(\delta \tilde{a}_{1}^{(s)} \mathfrak{D}^{(3)} u+\delta a_{0}^{(s)} u, v\right)_{\left[L^{2}(D)\right]^{m}}\right| \leq \tilde{M}\|u\|_{+, \gamma, \mathfrak{D}^{(3)}}\|v\|_{+, \gamma, \mathfrak{D}(3)}$

for all $u, v \in\left[H^{1}(D, S \cup S)\right]^{m}$ with a constant $0<\tilde{M}<1$, being independent on $u$ and $v$ then problem (3.17) is a Fredholm one.

\section{The spectral properties of the MiXed Problems}

In this section we use Theorems $3.13,3.14$ and the standard tools of Functional Analysis for the description of the completeness of the root elements of the mixed problem (3.17) in the spaces $H_{\mathfrak{D}}^{+, \gamma}(D),\left[H^{0, \gamma}(D)\right]^{m}$ and $H_{\mathfrak{D}}^{-, \gamma}(D)$. We study both the coercive and the non-coercive cases.

With this aim we consider the sesquilinear form

$$
(u, v)_{-, \gamma, \mathfrak{D}}:=\left\langle L_{0}^{-1} u, v\right\rangle_{\gamma} \text { for } u, v \in H_{\mathfrak{D}}^{-, \gamma}(D),
$$

on the space $H_{\mathfrak{D}}^{-, \gamma}(D)$. It is well known that $\sqrt{(u, u)_{-, \gamma, \mathfrak{D}}}=\|u\|_{-, \gamma, \mathfrak{D}}$ for all $u \in H_{\mathfrak{D}}^{-, \gamma}(D)(D)$. From now on we endow the space $H_{\mathfrak{D}}^{-, \gamma}(D)$ with the scalar product $(\cdot, \cdot)_{-, \gamma, \mathfrak{D}}$.

We recall that a compact self-adjoint operator $C$ is said to be of finite order if there is $0<p<\infty$, such that the series $\sum_{\nu}\left|\lambda_{\nu}\right|^{p}$ converges where $\left\{\lambda_{\nu}\right\}$ is the system of eigenvalues of the operator $C$ (here the summation is done counting the multiplicities of the eigenvalues, see, for instance, [13] and elsewhere).

Theorem 4.1. If $H_{\mathfrak{D}}^{+, \gamma}(D)$ is continuously embedded into $H^{0, \gamma}(D)$ then the inverse $L_{0}^{-1}$ of the operator given by (3.16) induces positive self-adjoint operators

$$
\begin{aligned}
\iota^{\prime} \iota L_{0}^{-1}: H_{\mathfrak{D}}^{-, \gamma}(D) \rightarrow & H_{\mathfrak{D}}^{-, \gamma}(D), \quad \iota L_{0}^{-1} \iota^{\prime}:\left[H^{0, \gamma}(D)\right]^{m} \rightarrow\left[H^{0, \gamma}(D)\right]^{m}, \\
& L_{0}^{-1} \iota^{\prime} \iota: H_{\mathfrak{D}}^{+, \gamma}(D) \rightarrow H_{\mathfrak{D}}^{+, \gamma}(D),
\end{aligned}
$$

which have the same systems of eigenvalues and eigenvectors; besides, the eigenvalues are positive. Moreover, if $H_{\mathfrak{D}}^{+, \gamma}(D)$ is continuously embedded into $H^{s, \gamma}(D)$ with $0<s \leq 1$ then they are compact operators of finite orders and there are orthonormal basis in the spaces $H_{\mathfrak{D}}^{+, \gamma}(D),\left[H^{0, \gamma}(D)\right]^{m}$ and $H_{\mathfrak{D}}^{-, \gamma}(D)$.

Proof. The first part of the theorem is well-known (see, for instance, [20], 21], 24]). Besides,

$$
\begin{gathered}
\left(\iota^{\prime} \iota L_{0}^{-1} u, v\right)_{-, \gamma, \mathfrak{D}}=\left(\iota L_{0}^{-1} u, \iota L_{0}^{-1} v\right)_{\left[H^{0, \gamma}(D)\right]^{m}},\left(L_{0}^{-1} \iota^{\prime} \iota u, v\right)_{+, \gamma, \mathfrak{D}}=(\iota u, \iota v)_{\left[H^{0, \gamma}(D)\right]^{m} .} \\
\left(\iota L_{0}^{-1} \iota^{\prime} u, v\right)_{\left[H^{0, \gamma}(D)\right]^{m}}=\left(L_{0}^{-1}\left(\iota^{\prime} u\right), L_{0}^{-1}\left(\iota^{\prime} v\right)\right)_{+, \gamma, \mathfrak{D}} .
\end{gathered}
$$

Moreover, Lemma 2.2 implies that under the hypothesis of the lemma, the operator $\iota$ is compact. Therefore the statement on the basis follows from the Hilbert-Schmidt theorems and the identities (4.1). That is why it is left to prove the statement on operator's orders only.

For the usual Sobolev spaces the statement follows from results of [1] (see also 24. Theorem 3.2]), because in this situation the operator $\iota^{\prime} \iota L_{0}^{-1}$ maps, in fact, $\left[H^{-s}(D)\right]^{m} \subset H_{\mathfrak{D}^{(j)}}^{-, \gamma}(D)$ to $H_{\mathfrak{D}^{(j)}}^{+, \gamma}(D) \subset\left[H^{s}(D)\right]^{m}, j=1,2$.

Since the embedding $H^{s, 0}(D) \rightarrow H^{s}(D)$ is obviously bounded, then for the weighted Sobolev spaces the correspondence $u \mapsto \rho^{-\gamma} u$ induces a continuous map 
$S^{+}: H^{s, \gamma}(D) \rightarrow H^{s}(D)$, and the correspondence $v \mapsto \rho^{\gamma} v$ induces a continuous map $S^{-}: H^{-s}(D) \rightarrow H^{-s, \gamma}(D)$. Hence, if the embedding $i_{s}: H^{+, \gamma}(D) \rightarrow$ $H^{s, \gamma}(D)$ is continuous then the results of [1] imply that the order of the operator $\iota_{s}^{\prime} \iota_{s} S^{+} i_{s} L_{0}^{-1} i_{s}^{\prime} S^{-}: H^{-s}(D) \rightarrow H^{-s}(D)$ equals to $2 s$ and it has the same eigenvalues as the operator $\iota^{\prime} \iota L_{0}^{-1}$ (here $\iota_{s}: H^{s}(D) \rightarrow L^{2}(D)$ is the natural embedding.

It is not difficult to show that the operator $L: H_{\mathfrak{D}}^{+, \gamma}(D) \rightarrow H_{\mathfrak{D}}^{-, \gamma}(D)$ induces a closed densely defined linear operator $T: H_{\mathfrak{D}}^{-, \gamma}(D) \rightarrow H_{\mathfrak{D}}^{-, \gamma}(D)$ with the domain $H_{\mathfrak{D}}^{+, \gamma}(D)$. The the operator $L_{0}$ corresponds to a symmetric closed operator $T_{0}: H_{\mathfrak{D}}^{-, \gamma}(D) \rightarrow H_{\mathfrak{D}}^{-, \gamma}(D)$ having the same eigenvectors as the operator $\iota^{\prime} \iota L_{0}^{-1}: H_{\mathfrak{D}}^{-, \gamma}(D) \rightarrow H_{\mathfrak{D}}^{-, \gamma}(D)$. As it is known, non-selfadjoint operators in infinitedimensional spaces may have not enough eigenvectors to form a basis. Hence the notion of the root vectors is very important.

Recall that a non-zero vector $u$ from the domain $D(T)$ of a linear operator $T$ on a linear space $H$ is called a root vector (or, the generalized eigenvector) for $T$, if there are numbers $N \in \mathbb{N}$ and $\lambda \in \mathbb{C}$ satisfying $(T-\lambda I)^{N} u=0$, where $I: H \rightarrow H$ is the identity operator in $H$.

The conditions providing the completeness of the root vectors are well known in the frames of the functional analysis (see [4, [5], [13], [14] and others).

Corollary 4.2. Under the hypotheses of Theorem 3.13, if $M<\sin \pi / m$ then the system of the root vectors of the closed operator $T$ is complete in the spaces $H_{\mathfrak{D}^{(j)}}^{-, \gamma}(D),\left[H^{0, \gamma}(D)\right]^{m}$ and $H_{\mathfrak{D}^{(j)}}^{+, \gamma}(D), j=1,2$. Moreover, for any $\delta>0$ all the eigenvalues of $T$ (except a finite number of them) belong to the angle $|\arg \lambda|<$ $\delta+\arcsin M$ in $\mathbb{C}$.

Proof. Follows from Theorems 3.13, 4.1 and the Spectral Theory of non-selfadjoint operators (see, for instance, [4, [5, 7], 24, Theorem 6.8]).

Corollary 4.3. Under the hypotheses of Theorem 3.14, if $\tilde{M}<\sin \pi / 2 m, H_{\mathfrak{D}\left({ }^{(3)}\right.}^{-, \gamma}(D)$, $\left[H^{0, \gamma}(D)\right]^{m}$ and $H_{\mathfrak{D}^{(3)}}^{+, \gamma}(D)$. Moreover, for any $\delta>0$ all the eigenvalues of $T$ (except a finite number of them) belong to the angle $|\arg \lambda|<\delta+\arcsin \tilde{M}$ in $\mathbb{C}$.

Proof. Follows from Theorems 3.14, 4.1 and the Spectral Theory of non-selfadjoint operators (see, for instance, [24, Theorem 4.5]).

Example 4.4. Let $\rho \equiv 1$. The mixed problem (3.17) for $A=\left(\mathfrak{D}^{(1)}\right)^{*} \mathfrak{D}^{(1)}$ and $B=\chi_{S}+\chi_{\partial D \backslash S} \sigma$ is classical in the Elasticity Theory (see [12, §12]); here $\chi_{M}$ is the characteristic function of the set $M$. As the corresponding sesquilinear form $(\cdot, \cdot)_{+, \gamma, \mathfrak{D}^{(1)}}$ is coercive for $b_{0,0}=0, \mu \geq \kappa>0, \lambda \geq 0$ (see Lemma 3.3), we may also consider the boundary operators $\chi_{S}+\chi_{\partial D \backslash S}\left(\sigma+T(x) \partial_{\tau_{0}}+\delta b_{0}\right)$ with a matrix $T$ having small entries of the class $C^{0, \alpha}(\partial D \backslash S), 1 / 2<\alpha \leq 1$ and with the perturbation $\delta b_{0}$ described in Theorem 3.13 . The low order acceptable perturbations are also indicated in Theorem 3.13. The completeness conditions are described in Corollary 4.2 .

Example 4.5. Let $D$ be the unit circle in $\mathbb{R}^{2}(\cong \mathbb{C})$, ans $S$ be that part of its boundary where $\arg (z) \in[0,2 \pi] \backslash[-\pi / 2, \pi / 2]$. For $\rho \equiv 1$, consider mixed problem (3.17) with $A=\left(\mathfrak{D}^{(3)}\right) * \mathfrak{D}^{(3)}$ and $B=\chi_{S}+\chi_{\partial D \backslash S}\left(\nu_{\mathfrak{D}^{(3)}}+b_{0,0}\right)$, see (3.1), (3.4). Let $\mu \geq \kappa>0, \mu+\lambda \geq 0$ be constants and $b_{0,0}>0$ be a matrix with constant entries. Let $\varphi \in C^{\infty}(\bar{D})$ equal to zero identically in a neighborhood of $S$ and 
equal to one on the part where $\arg (z) \in[-\pi / 4, \pi / 4]$. It is clear that the function $u_{\varepsilon}=\phi(z)\left(\Re v_{\varepsilon}, \Im v_{\varepsilon}\right)$, where

$$
v_{\varepsilon}(z)=\sum_{\nu=0}^{\infty} \frac{\bar{z}^{4 \nu}}{(4 \nu+1)^{(1+\varepsilon) / 2}},
$$

belongs to $H^{+, \gamma}(D)$ but for any $s \in(1 / 2,1]$ there is $\varepsilon>0$ such that $u_{\varepsilon} \notin H^{s}(D)$ (cf. [23, Examples 1,2]). Thus, $H_{\mathcal{D}^{(3)}}^{+, \gamma}(D)$ is continuously embedded into $\left[H^{1 / 2}(D)\right]^{2}$ (see Theorem [3.6), but it is not embedded into $\left[H^{s}(D)\right]^{2}$ for any $s \in(1 / 2,1]$ (cf. [23. Examples 1,2]). Moreover, as $Y \subset \partial S$, then this example can be easily adopted to the weighted spaces.

Clearly, $\nu_{\mathfrak{D}(3)}$ is responsible not for the stress/viscosity on the boundary but for a more large class of interactions with $\partial D$. For instance, interpreting the Lamé system as a linearization of the stationary version of the Navier-Stokes' type equations for the compressible fluids, we see that the boundary operator $\left(\nu_{\mathcal{D}(3)}+b_{0,0}\right)$ reflects rather the vorticity and the source density on conormal directions to $\partial D \backslash S$. This means that the boundary operator $\nu_{\mathfrak{D}^{(3)}}$ is more fit to study problems, related to models with the turbulent flows, than the operators $\nu_{\mathfrak{D}^{(1)}}$ and $\nu_{\mathfrak{D}^{(2)}}$. Then it is natural that the class of the possible solutions to (3.17) extends up to $H_{\mathcal{D}^{(3)}}^{+, \gamma}(D)$ due to the loss of the regularity of solutions near $\partial D \backslash S$.

Example 4.6. Let $\rho \equiv 1$. Consider mixed problem (3.17) for $A=\left(\mathfrak{D}^{(2)}\right)^{*} \mathfrak{D}^{(2)}$ and $B=\chi_{S}+\chi_{\partial D \backslash S}\left(\nu_{\mathfrak{D}^{(2)}}+h \mu(x) \partial_{\tau_{0}}\right)$ with a small parameter $h$, see (3.1), (3.4), in the case where $\mu \geq \kappa>0, \mu+\lambda \geq \kappa>0$. In particular, if we choose the vectors (3.18) as a basis among the tangential vectors to $\partial D$ then $d_{j}(x)=h \chi_{\partial D \backslash S} \mu(x) I_{m}$, $1 \leq j \leq m-1$.

Assume that $\mu, \lambda \in C^{0,1}(\bar{D})$. Then $\mu \in L^{\infty}(D), \nabla_{m} \mu \in L^{\infty}(D), \mu \in C^{0, \alpha}(\partial D \backslash$ $S$ ) for all $1 / 2<\alpha \leq 1$. According to Lemma 3.3, the norms of the spaces $H_{\mathfrak{D}^{(2)}}^{+, \gamma}(D)$ and $\left[H^{1}(D)\right]^{m}$ are equivalent. Lemma 3.10 implies that the first order terms induce the compact operators, acting from $\left[H^{1}(D)\right]^{m}$ to $\left[H^{-1}(D)\right]^{m}$. If the value $|h|$ is sufficiently small then problem (3.17) is a Fredholm one and its root vectors are dense in $\left[H^{1}(D)\right]^{m},\left[H^{-1}(D)\right]^{m},\left[L^{2}(D)\right]^{m}$. If the value $|h|$, is sufficiently small then problem (3.17) is uniquely solvable. The other acceptable perturbations are described in Theorem 3.13 .

If the coefficients $\lambda, \mu$ are constants then Gauß-Ostrogradskii formula implies

$\left|\left(\partial_{\tau_{0}} u, v\right)_{L^{2}(\partial D \backslash S) \mid}=\right| \sum_{j=1}^{m}\left(\nabla_{m} u_{j}, \partial_{j} v\right)_{L^{2}(D)}-\left(\operatorname{div}_{m} u, \operatorname{div}_{m} v\right)_{L^{2}(D)} \mid$ for all $u, v \in H^{1}(D, S)$,

i.e. $\mu\left\|\partial_{\tau_{0}}\right\| \leq 1$. But it follows from (3.5) that $\nu_{\mathfrak{D}^{(3)}}+b_{0,0}=\nu_{\mathfrak{D}^{(2)}}+b_{0,0}-\mu \partial_{\tau_{0}}$. Thus, if $\mu\left\|\partial_{\tau_{0}}\right\|<1$ then, for matrices $b_{0,0}$ with rather small entries, the mixed problem with the boundary operator $\nu_{\mathfrak{D}(3)}+b_{0,0}$ can be interpreted as a small perturbation of the mixed problem with the boundary operator $\nu_{\mathfrak{D}^{(2)}}$. However this contradicts with Example 4.6 because the space $H_{\mathfrak{D}^{(3)}}^{+, \gamma}(D)$ is not embedded into $H_{\mathcal{D}^{(2)}}^{+, \gamma}(D)=\left[H^{1, \gamma}(D)\right]^{m}$. Hence for constant Lamé coefficients the perturbation method is valid with $|h|<1$. In particular, formula (3.5) means that the mixed problem with the boundary operator $\chi_{S}+\chi_{\partial D \backslash S} \nu_{\mathfrak{D}^{(2)}}$ can not be investigated as the perturbation of the mixed problem with the boundary operator $\chi_{S}+\chi_{\partial D \backslash S} \sigma$ in the space $\left[H^{1, \gamma}(D)\right]^{m}$. 
In conclusion, we give examples of proper weight-functions.

Example 4.7. Consider the cylinder $D=\left\{\left(x_{1}, \ldots, x_{m-1}\right) \in \Omega, 0<x_{m}<1\right\}$ with the base $S=\left\{\left(x_{1}, \ldots, x_{m-1}\right) \in \Omega, x_{m}=0\right\}$ and the set $Y=\partial S$, where $\Omega$ is domain with smooth boundary in $\mathbb{R}^{m-1}$. Let $\phi\left(x_{1}, \ldots, x_{m-1}\right)$ be the defining function for the domain $\Omega$, i.e. it is a real-valued function with $\nabla \phi=1$ on $\partial \Omega$ such that $\Omega=\left\{\left(x_{1}, \ldots, x_{m-1}\right) \in \mathbb{R}^{m-1}: \phi\left(x_{1}, \ldots, x_{m-1}\right)<0\right\}$. Then $\rho(x)=$ $\sqrt{\phi^{2}\left(x_{1}, \ldots, x_{m-1}\right)+x_{m}^{2}}$.

Example 4.8. Consider the cube $D=\left\{-1<x_{j}<1,1 \leq j \leq m-1,0<x_{m}<1\right\}$ with a distinguished side $S=\left\{-1<x_{1}<1,-1<x_{2}<1,-1<x_{j}<1,1 \leq j \leq\right.$ $\left.m-1, x_{m}=0\right\}$ and the set $Y=\partial S$. In this situation we may set

$$
\rho(x)=\left(\Pi_{j=1}^{m-1}\left(\left(x_{j}-1\right)^{2}+x_{m}^{2}\right)\left(\left(x_{j}+1\right)^{2}+x_{m}^{2}\right)\right)^{1 / 2} .
$$

The work was supported by RFBR grants 14-01-00544 and 14-01-00081.

\section{REFERENCES}

[1] S. Agmon, On the eigenfunctions and on the eigenvalues of general elliptic boundary value problems, Comm. Pure Appl. Math. 15, pp. 119-147 (1962).

[2] S. Agmon, A. Douglis and L. Nirenberg, Estimates near the boundary for solutions of elliptic partial differential equations satisfying general boundary conditions, Part 1. Comm. Pure Appl. Math. 12, pp. 623-727 (1959).

[3] M. S. Agranovich, Elliptic operators on closed manifold, in: Current Problems of Mathematics, Fundamental Directions, Vol. 63, (VINITI, Moscow, 1990), pp. 5129.

[4] M. S. Agranovich, Spectral Problems in Lipschitz Domains, in: Modern Mathematics, Fundamental Directions, Vol. 39 (VINITI, Moscow, 2011), pp. 11-35.

[5] M. S. Agranovich, Strongly elliptic second order systems with boundary conditions on a non-closed Lipschitz surface, Funct. Anal. Appl. 45:1, pp. 115 (2011).

[6] M. Borsuk and V.A. Kondrat'ev, Elliptic Boundary Value Problems of Second Order in Piecewise Smooth Domains (Elsevier, Amsterdam-London, 2006).

[7] F. E. Browder, On the spectral theory of strongly elliptic differential operators, Proc. Nat. Acad. Sci. USA 45, pp. 1423-1431 (1959).

[8] S. Campanato, Sui problemi al contorno per sistemi di equazioni differenziale lineari del tipo dell'elasticitá, Ann. della Scuola Norm. Superiore, Cl. di Sci, Ser. III, 13:2, pp. 223-258 (1959).

[9] S. Campanato, Proprietá di taluni spazi di distribuzioni e loro applicazione, Ann. della Scuola Norm. Superiore, Cl. di Sci, Ser. III, 14:4, pp. 363-376 (1960).

[10] Yu. Egorov, V. Kondratiev, B.-W. Schulze, Completeness of eigenfunctions of an elliptic operator on a manifold with conical points, Russ. J. Math. Phys. 8:3, pp. 267-274 (2001).

[11] G. I. Eskin, Boundary Value Problems for Elliptic Pseudodifferential Operators (Nauka, Moscow, 1973).

[12] G. Fichera, Existence Theorems in Elasticity, in: Festkörpermechanik/Mechanics of Solids, edited by S. Flügge, C.A. Truesdell, Handbuch der Physik (Berlin-eidelbergNew York, Springer-Verlag, 1972), pp. 347389.

[13] I. Ts. Gokhberg and M. G. Krein, Introduction to the Theory of Linear Nonselfadjoint Operators in Hilbert Spaces, (AMS, Providence, R.I., 1969).

[14] M. V. Keldysh, On the characteristic values and characteristic functions of certain classes of non-selfadjoint equations, Dokl. AN SSSR 77, pp. 11-14 (1951).

[15] J.J. Kohn and L. Nirenberg, Non-coercive boundary value problems. Comm. Pure Appl. Math. 18, pp. 443-492 (1965).

[16] V. A. Kondrat'ev, Completeness of the systems of root functions of elliptic operators in Banach spaces, Russ. J. Math. Phys. 6:10, pp. 194-201 (1999).

[17] V. A. Kondrat'ev, Boundary problems for parabolic equations in closed domains, Trans. Moscow Math. Soc. 15, pp. 400-451 (1966). 
[18] O. A. Ladyzhenskaya, and N. N. Uraltseva, Linear and Quasilinear Equations of Elliptic Type (Nauka, Moscow, 1973).

[19] L.D. Landau and E.M. Lifshitz, Fluid Mechanics, Volume 6 of A Course of Theoretical Physics (Pergamon Press, London-New York-Paris, 1959).

[20] J.L. Lions and E. Magenes, Non-Homogeneous Boundary Value Problems and Applications, Vol. 1 (Springer-Verlag, Berlin-Heidelberg-New York, 1972).

[21] V. P. Mikhailov, Partial Differential Equations (Nauka, Moscow, 1976).

[22] M. Schechter, Negative norms and boundary problems, Ann. Math. 72:3, pp. 581-593 (1960).

[23] A. Shlapunov and A. Polkovnikov, On the spectral properties of a non-coercive mixed problem associated with $\bar{\partial}$-operator, J. Siberian Fed. Uni. 6:2, (2013).

[24] A. Shlapunov and N. Tarkhanov, On completeness of root functions of Sturm-Liouville problems with discontinuous boundary operators, Journal of Differential Equations 255, pp. 33053337 (2013).

[25] A. Shlapunov and N. Tarkhanov, Bases with double orthogonality in the Cauchy problem for systems with injective symbols, Proc. London Math. Soc. 71:3, pp. 1-52 (1995).

[26] A. Shlapunov, N. Tarkhanov, and B. W. Schulze, Green integrals on manifolds with cracks, Annals of Global Analysis and Geometry 24, pp. 131-160 (2003).

[27] N. Tarkhanov, On the root functions of general elliptic boundary value problems, Compl. Anal. Oper. Theory 1, pp. 115-141 (2006).

(Alexander Shlapunov) Siberian Federal University, Institute of Mathematics, Pr. Svobodnyi 79, 660041 Krasnoyarsk, Russia

E-mail address: ashlapunov@sfu-kras.ru

(Anastasiya Peicheva) Siberian Federal University, Institute of Mathematics, Pr. SvoBodnyi 79, 660041 Krasnoyarsk, Russia

E-mail address: peichevaas@mail.ru 